\title{
Inexpensive airfoil shape optimization for vertical axis wind turbine and its validation
}

\author{
Wataru YAMAZAKI* and Yuta ARAKAWA* \\ *Dept. of Mechanical Engineering, Nagaoka University of Technology \\ 1603-1 Kamitomioka-machi, Nagaoka, Niigata, 940-2188, Japan \\ E-mail: yamazaki@mech.nagaokaut.ac.jp
}

Received 6 July 2015

\begin{abstract}
In this research, an inexpensive aerodynamic shape optimization of airfoil is performed for Darrieus type vertical axis wind turbine (VAWT). Computational fluid dynamics (CFD) evaluations of various airfoil shapes are treated inexpensively by considering the apparent flow velocity magnitudes and apparent angles of attack at multiple positions of VAWT airfoil, which includes some assumptions as two dimensional steady flows and no consideration about flow interactions between blade airfoils. Global optimal shapes are efficiently explored by using a Kriging response surface model approach. An optimal VAWT airfoil is obtained by the maximization of a proposed objective function. Since our objective function for this design optimization of VAWT airfoil can be primary decomposed into the terms of thrust force and pitching moment, another multi-objective optimization of thrust/pitching moment coefficients is also performed for detailed discussions about the obtained optimal airfoil. Analysis of variance is performed on the Kriging response surface model to extract design knowledge in this design optimization problem. The obtained optimal airfoil achieves a significant improvement in the aerodynamic performance compared with an existing airfoil. Finally, expensive unsteady CFD simulations are performed for the obtained airfoils, that take into account unsteady flow interactions between three blade airfoils. The results of the unsteady CFD simulations validate the effectiveness of the proposed aerodynamic shape optimization approach for VAWT airfoil.
\end{abstract}

Key words : Vertical axis wind turbine, Darrieus rotor, Aerodynamic shape optimization, Airfoil, Design knowledge extraction, Kriging response surface

\section{Introduction}

As latest emergency power source, small wind turbines are watched with keen interest. Since the small wind turbines are useful for the construction of distributed power generation systems, the demand of the small wind turbines will increase for some time in the future. There are two main types of the wind turbines, that are the horizontal-axis wind turbine (HAWT) and vertical-axis wind turbine (VAWT). The former is the more conventional wind turbine whose axis of rotation is parallel to the main flow. The HAWT has been the main subject of wind turbine researches for decades. The latter has attracted much more attention in recent years, whose axis of rotation is perpendicular to the main flow. Many experimental/numerical investigations have been performed in the literature (Bah, et al., 2013, Carrigan, et al., 2012, Ferreira, et al., 2009, Greenblatt, et al., 2012, Han, et al., 2013, Howell, et al., 2010, Seki, 1991, Wakashima, et al., 2012, Wakashima, et al., 2013). The VAWT has an advantage over the HAWT in the flexibility about the direction of the main flow. Lower noise and safety advantage at gust conditions are also pointed out in the literature. There are two major types in VAWT that are the Darrieus rotor and Savonius rotor. Since the Darrieus rotor, which generates power due to the lift force produced by rotating airfoils, has larger power coefficient than the Savonius rotor in general, we focus on the Darrieus-type VAWT in this research.

For the development of efficient Darrieus-type VAWTs, the airfoil shape of the main blades is very important. The realistic flowfields around VAWTs are, of course, completely three-dimensional as well as unsteady. Although computational fluid dynamics (CFD) methods have reached a high level of maturity in terms of efficiency and 
robustness in the past years, three-dimensional unsteady CFD evaluations around full VAWT configurations are still computationally expensive, especially when its shape optimization is taken into consideration. As a matter of fact, at least one hundred times of performance evaluation for different airfoil shapes are essential in such airfoil shape optimizations.

In this research, therefore, we try to optimize the airfoil shape for VAWT inexpensively by proposing a simplified shape optimization problem based on 2D steady flow simulations. The simplified problem is defined by considering apparent flow velocity magnitudes and apparent angles of attack at multiple positions of VAWT airfoil. In the present optimization problem, the speed ratio between the uniform flow and rotation $\beta$, which is equivalent with the tip speed ratio, is specified to 0.7069 . Although general operational conditions of the Darrieus-type VAWT are $\beta>1$, this design condition is chosen to increase the aerodynamic performance of the Darrieus-type VAWT at lower rotational condition. This can contribute to obtain design knowledge to increase the performance of small VAWT, since its performance at lower rotational conditions is an important subject. In addition, flow separation / stall occurs periodically in this design condition due to its wide range of the apparent angle of attack (discussed in section 3), which gives a challenging optimization problem from the viewpoint of aerodynamic design of airfoil. A Kriging response surface model approach is utilized for the airfoil shape optimization to explore global optimal solutions. Finally, the validity of the obtained optimal configuration is investigated by more accurate unsteady flow simulations in this research. The present paper is organized as follows. The CFD / shape optimization methodologies used in this research are concisely described in section 2 . In section 3, the simplified aerodynamic shape optimization problem for VAWT airfoil is defined and then its results are discussed. The validity of the obtained optimal configuration is discussed in section 4. Finally, we provide concluding remarks in section 5.

\section{Computational methodologies}

In this section, computational methodologies used for the shape optimization of VAWT are introduced concisely.

\subsection{Optimization approach}

In this research, a surrogate model-based global design optimization method is utilized which makes use of a Kriging response surface model. An ordinary Kriging surrogate model (Forrester, et al., 2008, Jones, et al., 1998, Yamazaki, et al., 2013) is used to construct the surrogate models of aerodynamic functions in design variable space. Firstly, initial sample points are generated in the design variable space by a Latin hypercube sampling method, and then these are evaluated by CFD computations. By using the information of the initial sample points, initial surrogate models are constructed. The search of a promising location in the design variable space is executed by a real-coded multi-objective genetic algorithm (Fonseca, et al., 1993, Kim, et al., 2009) on the surrogate models. The promising location is explored by the criteria of expected improvement (EI) (Jones, et al., 1998). The function of EI expresses a potential for improvement in design variable space which considers both estimated function value as well as uncertainty of the surrogate model. The CFD computation is performed for the explored promising location (configuration) where EI is maximal, and then new surrogate models are created by adding its information. By the iterative process described above, the accuracies of the surrogate models are efficiently increased around the promising locations in the design variable space. The flowchart of this optimization process is shown in Fig.1.

The constructed Kriging model is also utilized to extract design knowledge information. In this research, an analysis of variance (ANOVA) is performed on the Kriging model to discuss the significance of each design variable to an aerodynamic performance function (Jones, et al., 1998).

\subsection{CFD method}

In this research, two-dimensional incompressible steady flow simulations are performed by a SIMPLE-based scheme. Since the turbulent intensity of natural wind is generally $10-20 \%$, we assume fully turbulent flows in this study, and then the Spalart-Allmaras one equation turbulence model is utilized in the present computation (Wakashima, et al., 2012). The computational grid topology utilized in this study is O-type, whose total number of grid points is about 30,000. The computational grid is visualized in Fig.2 whose airfoil configuration is NACA642A015. Its outer boundary is located at 10 chord lengths away from the airfoil. At the inlet, the flow velocity is fixed while a Neumann condition is given for the static pressure. At the outlet, the pressure is fixed while a Neumann condition is given for the flow velocity. Typical y+ values on this computational grid are about $2 \sim 3$. 
For the validation of the CFD approach for this research, solution accuracies not only at low (general) angles of attack, but also at high angles of attack are important since the apparent angle of attack of VAWT airfoil becomes high at some positions of the airfoil. Therefore, CFD computations should be compared with experimental data covering the whole range of the angle of attack from -180 to +180 degrees. The wind tunnel experimental data of NACA642A015 airfoil at the Reynolds number of $1.3 \times 10^{5}$ (Seki, 1991) is utilized for this validation. The experimental/numerical results are compared in Fig.3. It can be confirmed that all CFD aerodynamic coefficients, lift, drag and pitching moment at the quarter-chord location show qualitative agreements with the experimental data at the whole range of the angle of attack. This result indicates the sufficient accuracy of the present CFD method for the aerodynamic shape optimization of VAWT airfoil.

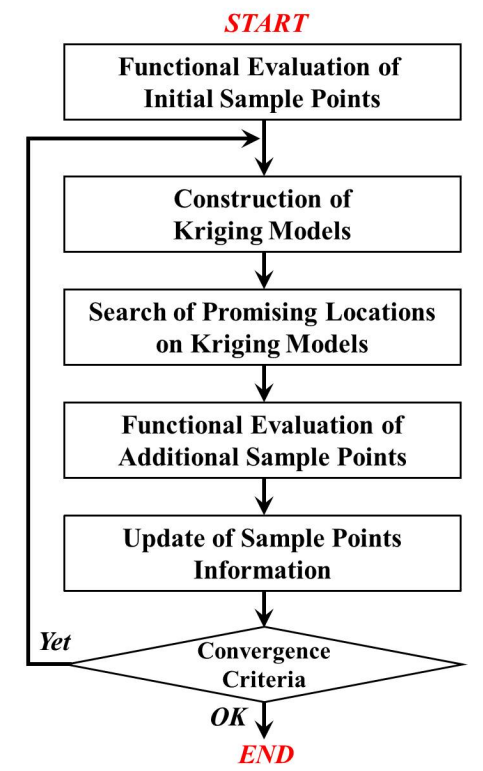

Fig.1 Flowchart of optimization via Kriging model.

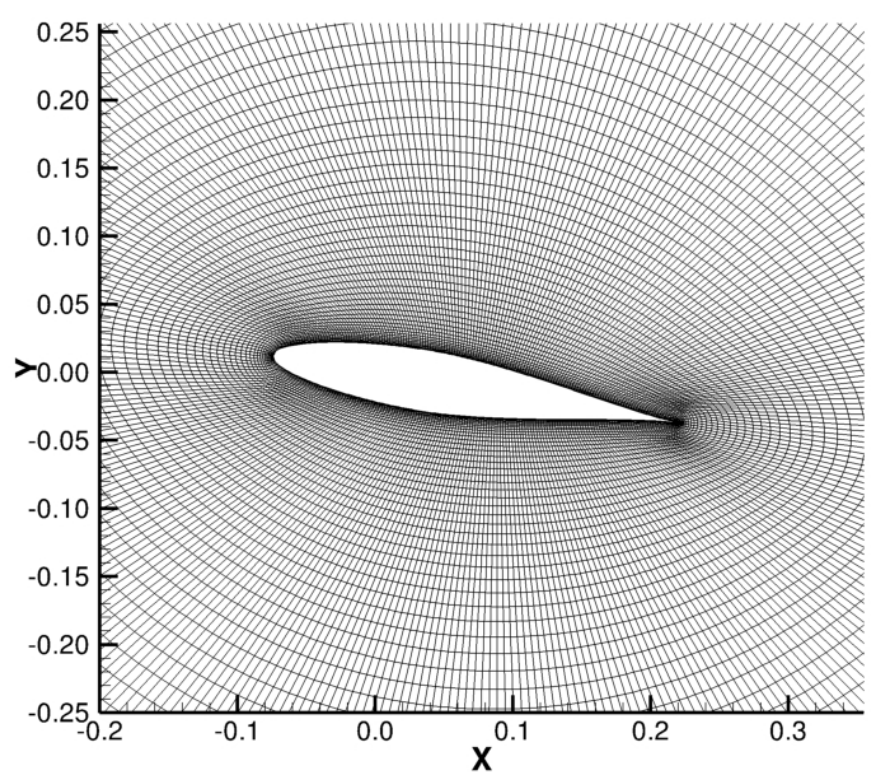

Fig.2 Computational grid around NACA642A015 airfoil.

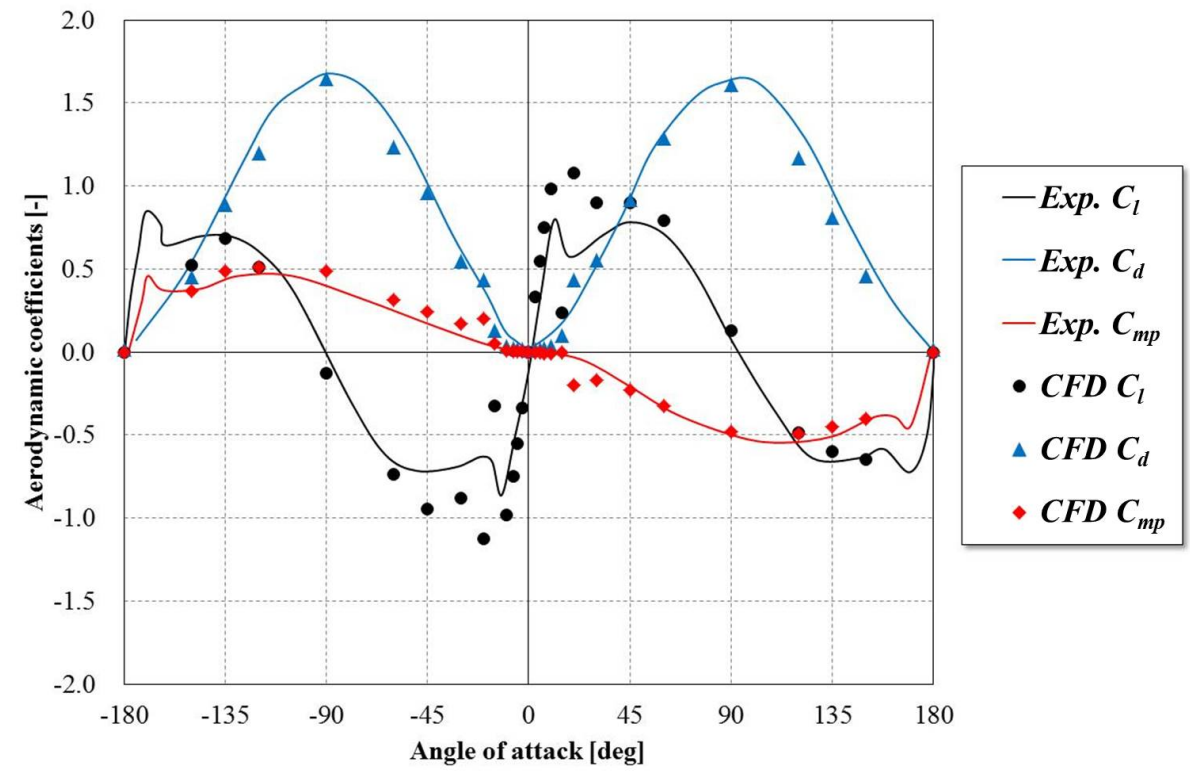

Fig.3 Comparison with experimental data of NACA642A015 airfoil, indicating the sufficient accuracy of the present CFD approach. 


\section{Aerodynamic shape optimization of VAWT airfoil}

In this section, the design optimization problem of VAWT airfoil is defined and then its results are discussed.

\subsection{Definition of inexpensive shape optimization problem for VAWT airfoil}

The schematic sketches of our considering condition are shown in Fig.4. The direction of uniform flow is assumed to be from left to right. The uniform flow velocity magnitude is defined by $U_{\infty}$. We consider only one airfoil of VAWT whose rotational velocity is defined by $\beta U_{\infty}$ where $\beta$ is the speed ratio between the uniform flow and rotation. By specifying $U_{\infty}$ and $\beta$, the apparent velocity magnitude as well as apparent angle of attack of the airfoil at any locations can be calculated by the composition of the two velocity vectors. The airfoil is assumed to rotate counterclockwise and then the rotational angle $\theta$ is defined as is shown in Fig.4. The relationships between the apparent velocity magnitude and apparent angle of attack at some settings of $\beta$ are indicated in Fig.5 in which the uniform flow velocity magnitude is fixed to $10 \mathrm{~m} / \mathrm{s}$. The apparent velocity magnitude is the highest at $\theta$ of 270 degrees while it is the lowest at $\theta$ of 90 degrees in arbitral cases. When $\beta$ is less than unit, the apparent angle of attack is varied from -180 to +180 degrees for one rotation. With the increase in $\beta$ over unit, on the other hand, the range of the apparent angle of attack narrows. In this study, aerodynamic performance of an airfoil is evaluated at ten degrees intervals of the rotational angle under the corresponding apparent velocity magnitude / angle of attack. Therefore, 36 steady CFD evaluations around an airfoil are required for an airfoil configuration, that can be executed in parallel. Then, averaged aerodynamic performance for one rotation is evaluated as follows:

$$
\begin{aligned}
& \bar{C}_{t}=\frac{1}{2 \pi} \int_{0}^{2 \pi} C_{t} d \theta \cong \frac{1}{36} \sum_{i=1}^{36} C_{t i}=\frac{1}{36} \sum_{i=1}^{36} \frac{F_{t i}}{q_{\infty} c} \\
& \bar{C}_{n}=\frac{1}{2 \pi} \int_{0}^{2 \pi} C_{n} d \theta \cong \frac{1}{36} \sum_{i=1}^{36} C_{n_{i}}=\frac{1}{36} \sum_{i=1}^{36} \frac{F_{n_{i}}}{q_{\infty} c} \\
& \bar{C}_{m p}=\frac{1}{2 \pi} \int_{0}^{2 \pi} C_{m p} d \theta \cong \frac{1}{36} \sum_{i=1}^{36} C_{m p_{i}}=\frac{1}{36} \sum_{i=1}^{36} \frac{M_{p_{i}}}{q_{\infty} c^{2}}
\end{aligned}
$$

where $q_{\infty}$ and $c$ are respectively the dynamic pressure at $U_{\infty}$ of $10 \mathrm{~m} / \mathrm{s}$ and chord length of the airfoil. $C_{t}, C_{n}$ and $C_{m p}$ are respectively the thrust force coefficient, normal force coefficient and pitching moment coefficient at the quarter-chord location. The thrust/normal force directions are defined as Fig.4, which is based on the tangential direction of the rotational motion. It is important to note that the thrust/normal force directions are different from the lift/drag force directions with respect to the apparent angle of attack. Finally, a performance function is defined as follows in this research:

$$
P=C_{m p}+C_{t} \frac{r}{c}-C_{n} \frac{c / 4}{c}
$$

where $r$ is the radius of VAWT. The airfoil is assumed to be vertically located to the radial direction at the $50 \%$ chord location, which gives the coefficient $c / 4$ in the third term of Eq.(4). This performance function indicates the rotational torque coefficient at the axis of rotation. The objective function of this study is finally defined as follows:

$$
\bar{P}=\bar{C}_{m p}+\bar{C}_{t} \frac{r}{c}-\bar{C}_{n} \frac{c / 4}{c}
$$

This function indicates the averaged rotational torque coefficient at the axis of rotation for one rotation, which can be evaluated only by 2D flow simulations around an airfoil. The maximization of this function will yield efficient airfoil configurations for VAWT although this approach includes assumptions as two dimensional steady flows and no consideration about the flow interactions between blade airfoils.

In this study, the uniform flow velocity is set to $10 \mathrm{~m} / \mathrm{s}$. The radius of VAWT and airfoil chord length are respectively set to $0.675 \mathrm{~m}$ and $0.3 \mathrm{~m}$. The rotational condition of the blade airfoil is set to $100 \mathrm{rpm}$, which results in $\beta$ of 0.7069 . Although general operational conditions of VAWT are $\beta>1$, this design condition is chosen to increase the aerodynamic performance of VAWT at lower rotational condition. In addition, flow separation / stall occurs periodically in this design condition due to the wider range of the apparent angle of attack, which gives a challenging design optimization problem.

Various airfoil shapes are defined by Bezier curves as shown in Fig.6. The upper/lower surfaces are separately defined by two Bezier curves that are based on seven control points. Since the vertical coordinates of the control points 
(except the fixed points at the leading/trailing edges) are treated as design variables, the total number of design variables is nine in this study, that are defined as $Y 1-Y 9$ in Fig.6. In this airfoil shape definition, $Y 1$ is utilized at both the upper and lower surfaces to define the rounded leading edge configurations whose major reason is to reduce the total number of design variables. The definition of $Y 9$ is also different from the other design variables, which is to satisfy positive thickness of the airfoil at the trailing edge region.

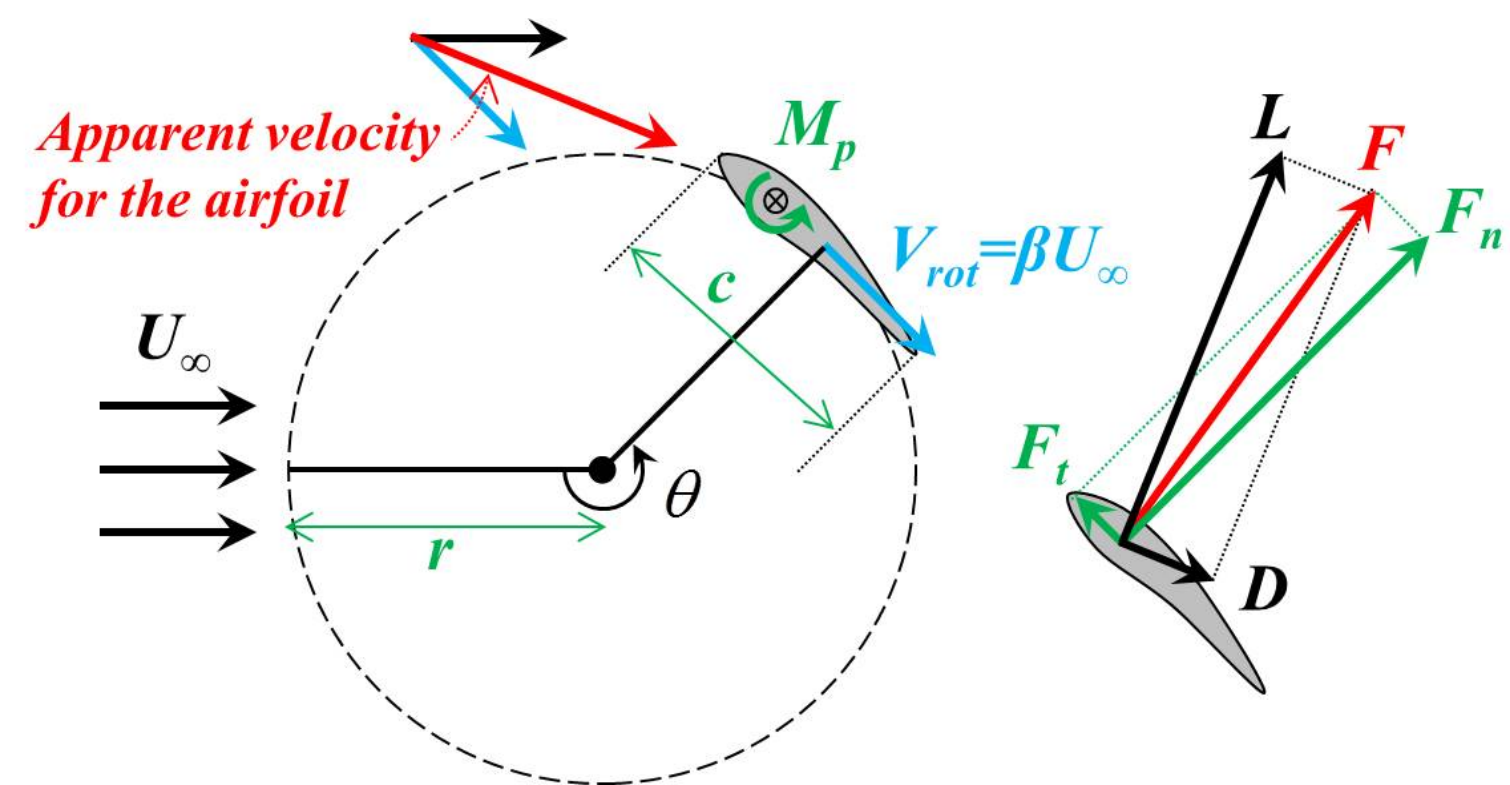

Fig.4 Schematic sketches of our design optimization problem definition of VAWT airfoil. Apparent velocity magnitude / angle of attack of an airfoil at rotational angle of $\theta$ are shown on the left-hand side. Thrust and normal force directions are defined on the right-hand side.

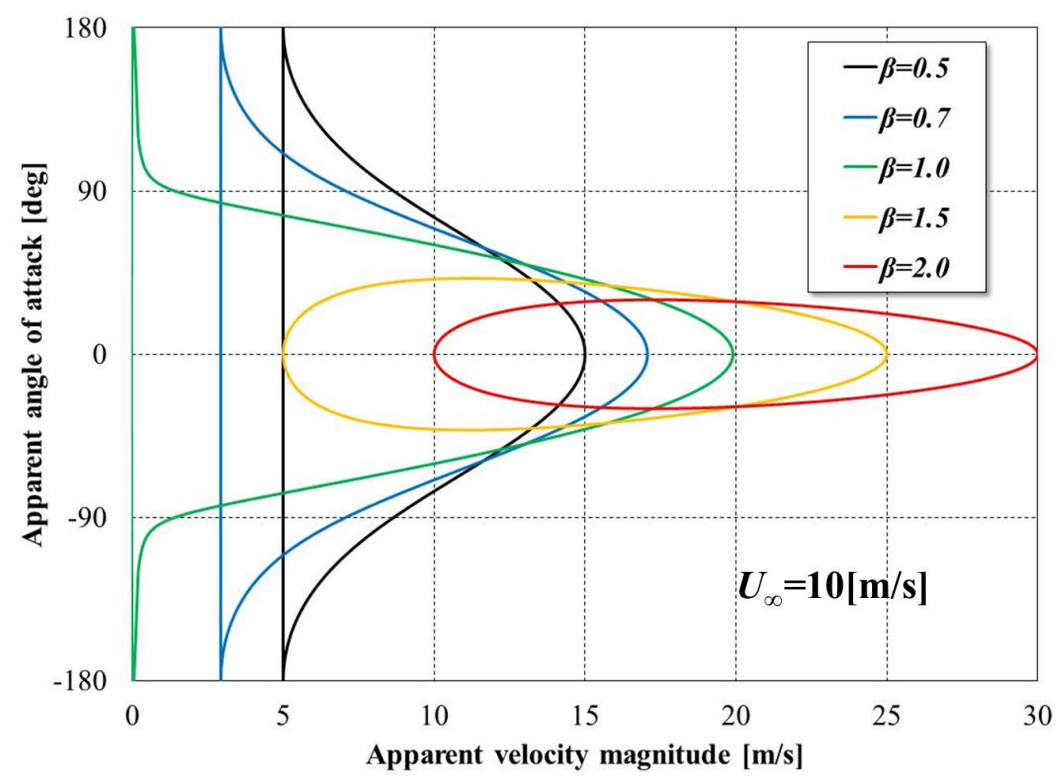

Black: Uniform flow

Blue: Rotation

Red: Composition

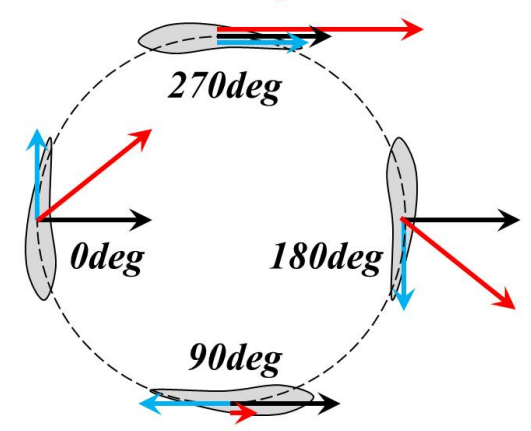

Fig.5 Relationships between apparent velocity magnitude and apparent angle of attack for one rotation. Schematic sketch is shown on the right-hand side. 


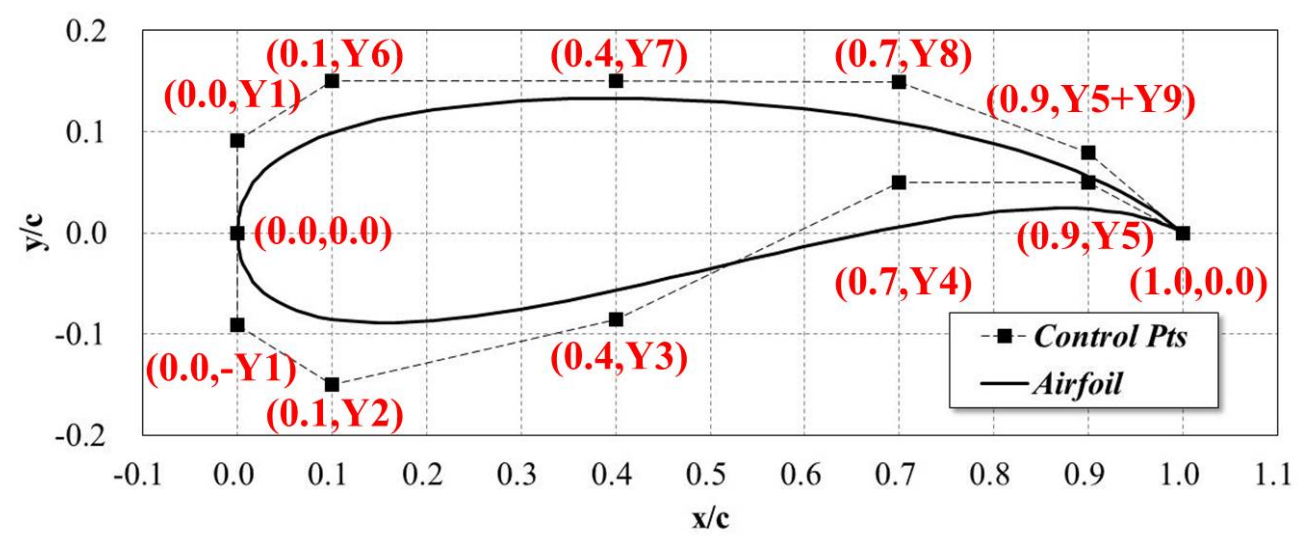

Fig.6 Airfoil shape parameterization via Bezier curves. Various airfoil shapes are generated by nine design variables. Vertical/horizontal axes are normalized by the chord length of airfoil.

\subsection{Results and discussion}

The number of initial sample points is set to 96, and then 50 additional sample points are iteratively selected/evaluated to maximize the objective function of Eq.(5) in this study. Since the first and second terms of Eq.(5) have dominant impact to the objective function of $\bar{P}$, the maximization of $\bar{C}_{t}$ and $\bar{C}_{m p}$ are also treated in another multi-objective optimization problem for detailed discussions. In Fig.7, the obtained non-dominated optimal solutions of the multi-objective optimization are compared with the optimal solution which was obtained by the maximization of Eq.(5). It can be seen that there is a tradeoff relationship between $\bar{C}_{t}$ and $\bar{C}_{m p}$. The performance of the obtained optimal solution via Eq.(5), which is named as design D, is almost comparable with that of a non-dominated solution which has beneficial performance in $\bar{C}_{t}$. This result implies the insignificance of the third term of Eq.(5) which expresses the effect of $\bar{C}_{n}$ in the present design optimization problem. It can be also confirmed that the obtained optimal solutions in these design optimizations achieve remarkable superiority over NACA642A015 airfoil. The aerodynamic performances of some representative optimal solutions (A D in Fig.7) are compared in Table 1.

The airfoil shapes of the representative optimal solutions are compared in Fig.8. It can be seen that the leading edge radiuses of the optimal airfoils are larger than the NACA642A015 airfoil. The optimal shape with respect to $\bar{C}_{t}$ (A) is almost a symmetrical airfoil with no camber, while the optimal shape with respect to $\bar{C}_{m p}(\mathrm{C})$ is a positive camber airfoil. The optimal shape with respect to $\bar{P}$ (D) is very comparable with the design of A except its positive camber at the trailing edge region. The distributions of the performance function $P$ for one rotation are compared in Fig.9. The increase in $P$ at the whole range of rotational angle can be seen in the optimal configuration of D compared with the NACA642A015 airfoil. The flow visualizations of the NACA642A015 airfoil and the representative optimal airfoils at $\theta$ of 240 and 300 degrees are shown in Fig.10 (Airfoil A is omitted since it is very similar with D). At the rotational angles, large differences in $P$ were observed in Fig.9. Significant differences of the flowfields can be observed between optimal airfoil D and other airfoils. The flow is massively separated with the NACA and C airfoils while the flow separation is suppressed with the optimal airfoil D mainly due to its larger leading edge radius.

To extract more engineering design information of VAWT airfoil, ANOVA is performed on the Kriging models with respect to $\bar{P}, \bar{C}_{t}$ and $\bar{C}_{m p}$. The obtained significances of all design variables are summarized in the pie charts of Fig.11. The tendencies of $\bar{P}$ and $\bar{C}_{t}$ are approximately equivalent, in which $Y 1, Y 2$ and $Y 6$ have primary significances. The interaction terms between $Y 1-Y 2$ and $Y 1-Y 6$ also have some significances in $\bar{P}$ and $\bar{C}_{t}$. These results indicate $\bar{P}$ and $\bar{C}_{t}$ are sensitive to the leading edge configuration as is confirmed from the definition of the design variables in Fig.6. On the other hand, $Y 4, Y 5, Y 8$ and $Y 9$ have primary significances in $\bar{C}_{m p}$, which implies $\bar{C}_{m p}$ is sensitive to the trailing edge configuration. Briefly saying, therefore, the leading edge configuration is important in this design optimization problem. By taking larger leading edge radius (shown in Fig.8), flow separation can be suppressed at several rotational angles (shown in Fig.10), which yields better aerodynamic performance in the optimal configuration (shown in Fig.9). For more sophisticated airfoil design in the present optimization problem of VAWT airfoil, more degree of freedom of the geometry definition around the leading edge may be required. 
Table 1 Summary of aerodynamic performance of optimal airfoils.

\begin{tabular}{llll}
\hline Airfoil shape & $\bar{P}$ & $\bar{C}_{t}$ & $\bar{C}_{m p}$ \\
\hline NACA642A015 & 0.0267 & 0.0119 & -0.0002 \\
A & 0.9484 & 0.4215 & 0.0003 \\
B & 0.7459 & 0.2990 & 0.2747 \\
C & 0.1705 & 0.0127 & 0.3264 \\
D & 0.9613 & 0.4172 & 0.0794 \\
\hline
\end{tabular}

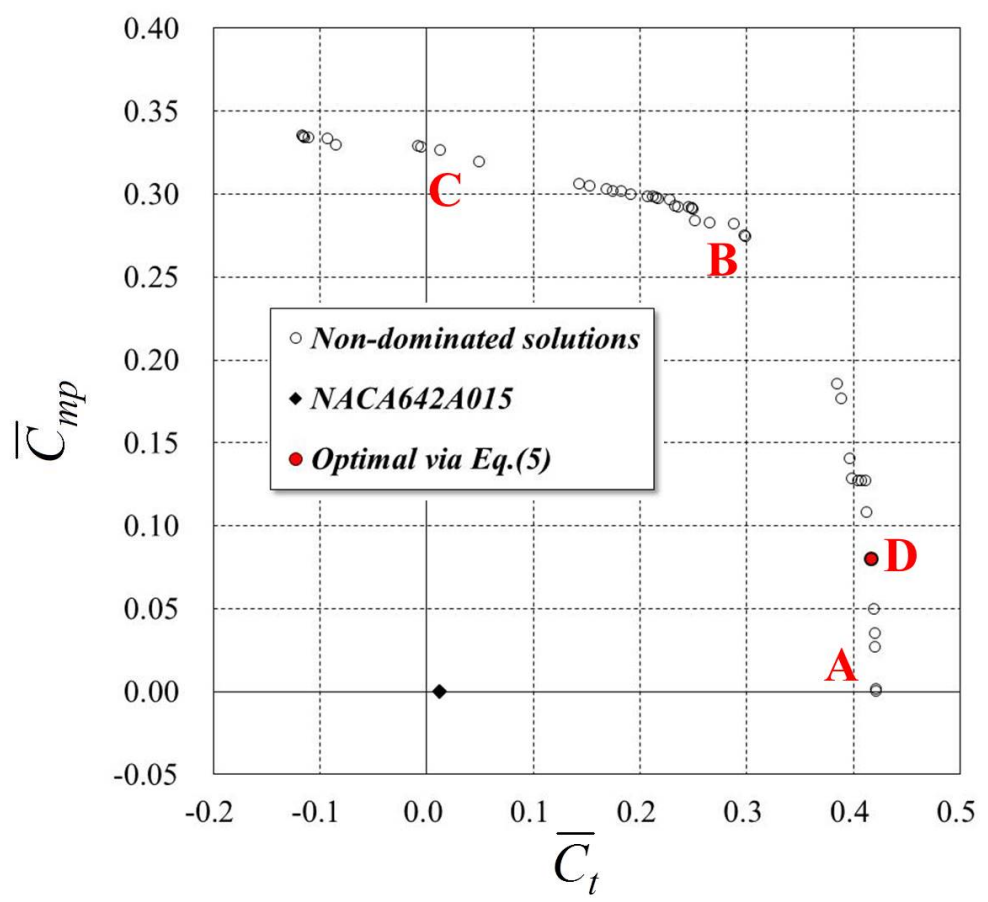

Fig.7 Comparison of obtained optimal solutions in $\bar{C}_{t}-\bar{C}_{m p}$ space. Designs A $\sim \mathrm{C}$ were obtained by solving a multi-objective optimization problem. Design D was obtained by maximizing the proposed objective function. 


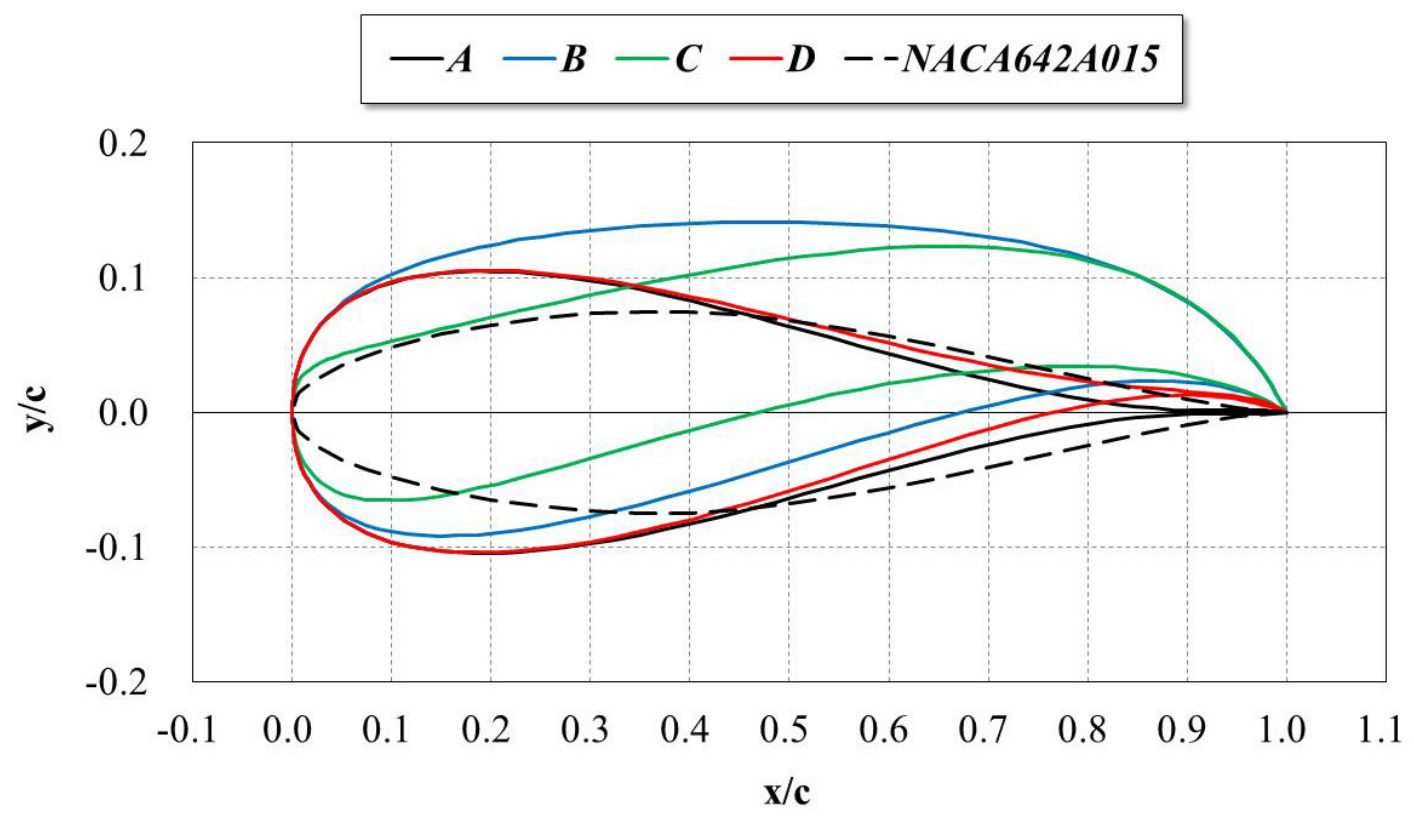

Fig.8 Airfoil shapes of representative optimal solutions. Vertical/horizontal axes are normalized by the chord length of airfoil. Note that the scales of $x, y$ coordinates are different for better visibility.

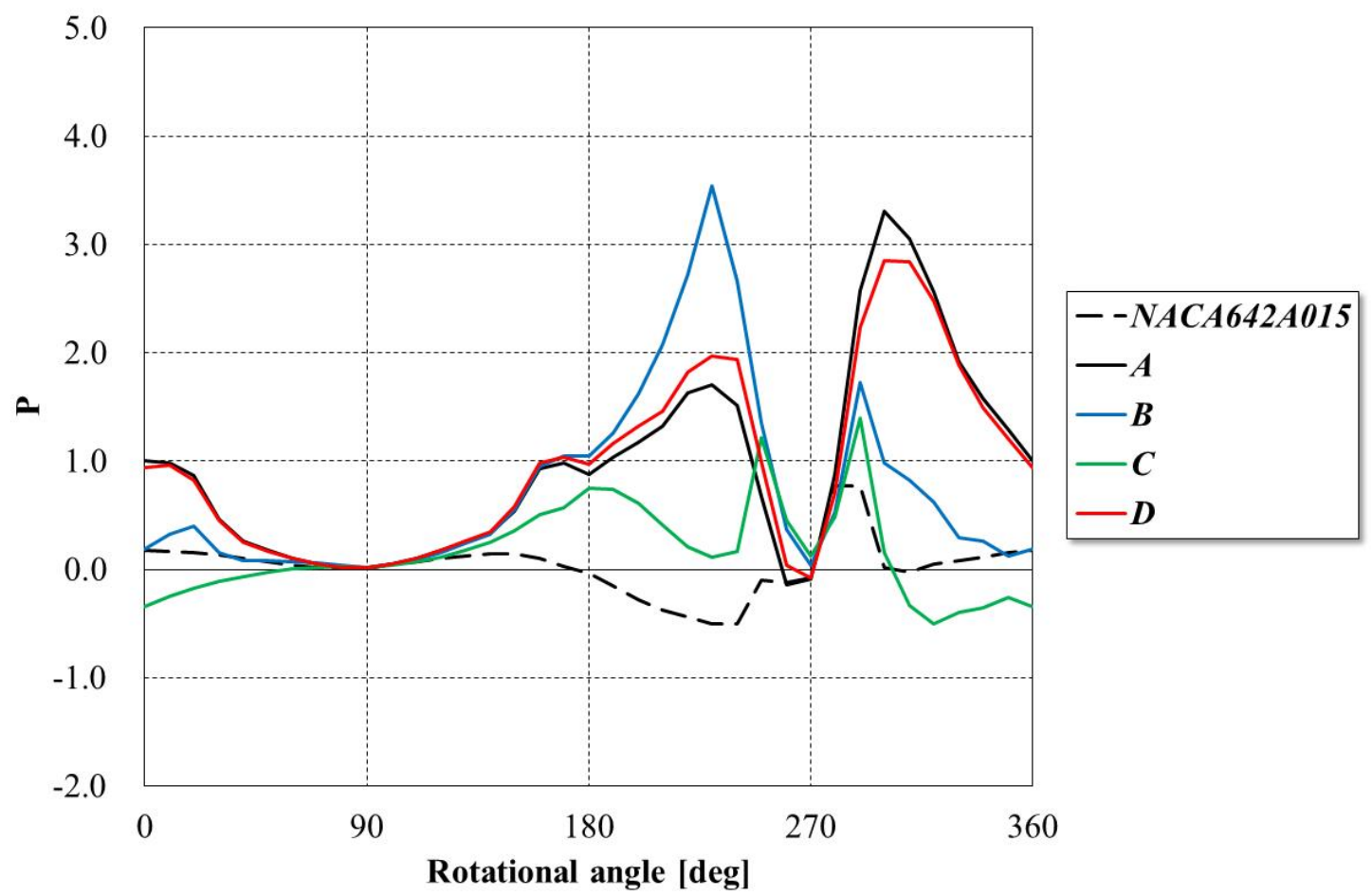

Fig.9 Performance function distributions of representative optimal solutions. 


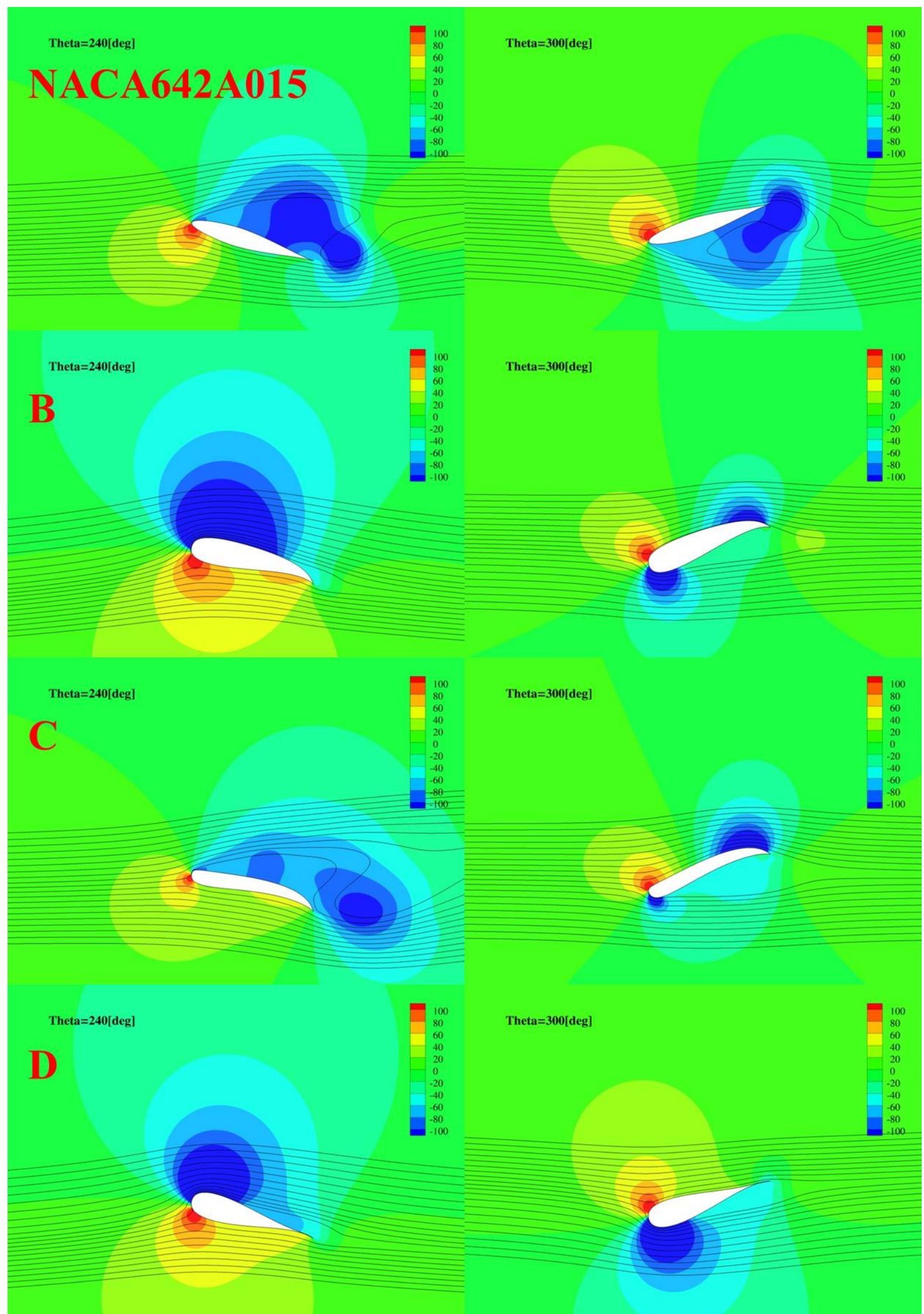

Fig.10 Visualizations of streamlines/pressure variation of representative optimal airfoils. The unit of pressure is the Pascal. Apparent flow direction is from left to right in all cases. Left: $\theta$ of 240 degrees, Right: $\theta$ of 300 degrees. 

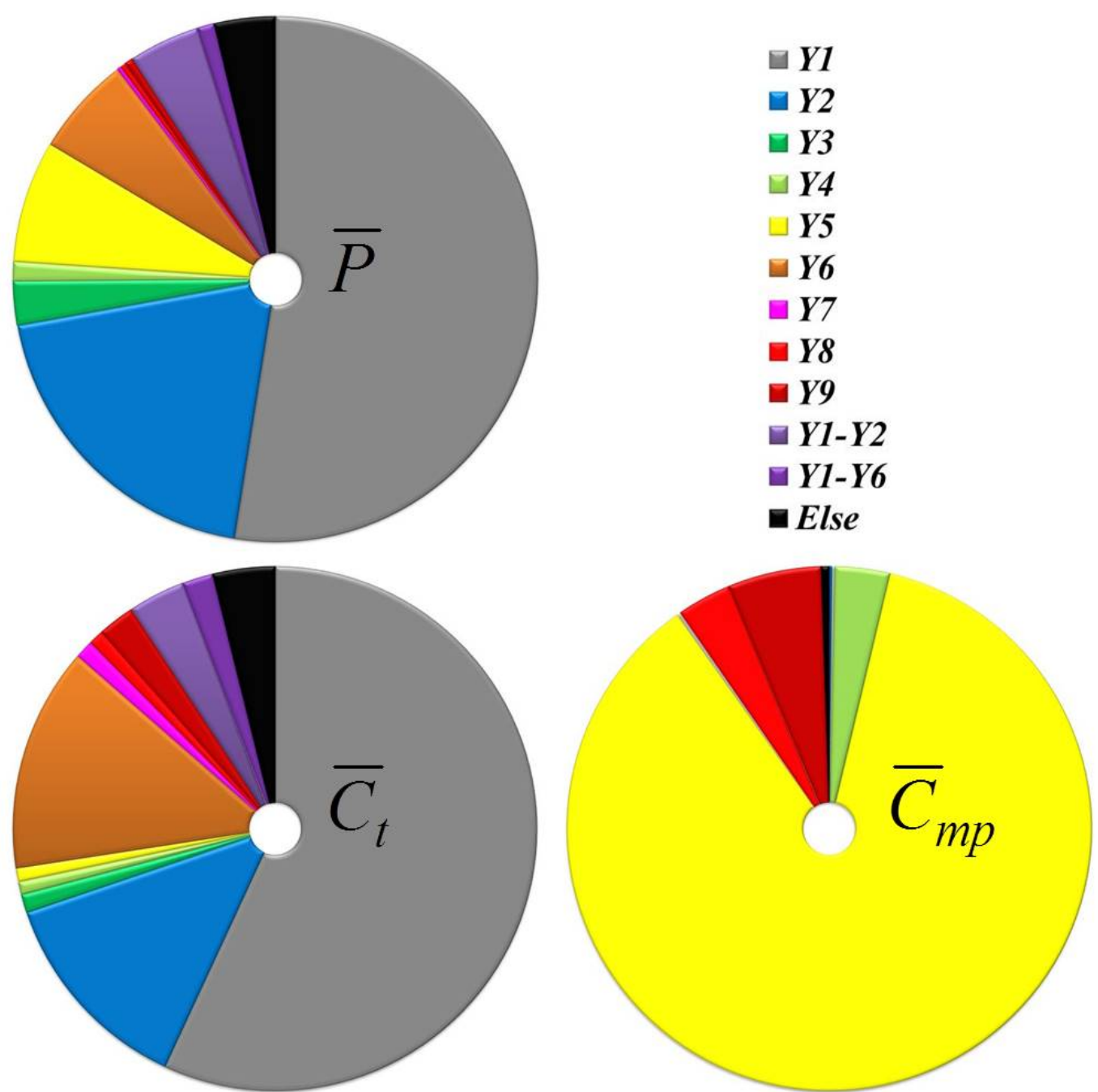

Fig.11 Significances of all design variables to representative performance functions. These pie charts were obtained by ANOVA on the obtained Kriging response surface model.

\section{Validity of obtained optimal airfoils / developed optimization approach}

In this section, more accurate numerical flow simulations are performed to validate the obtained optimal airfoil as well as the developed optimization approach of VAWT airfoil. The numerical simulation performed in this section is for 2D unsteady flows and taking into account the flow interactions between three blade airfoils of VAWT. The center pole of VAWT is also included in the computational domain. The whole computational domain is divided into two subdomains in these simulations. The three blade airfoils and the center pole are included in a (rotating) subdomain while its far-field region is defined by another (stationary) subdomain. The boundary of the two subdomains is treated by a sliding mesh approach. The whole computational domain is schematically shown in Fig.12. The present computations are performed using the commercial software package ANSYS CFX 13.0. Incompressible Reynolds-averaged Navier-Stokes equations are solved with a standard $k-\varepsilon$ turbulence model.

The unsteady flow simulations are performed with the NACA642A015 airfoil and the obtained representative airfoil configurations. In the flow simulations, the uniform flow velocity magnitude and rotational speed are respectively set to $10 \mathrm{~m} / \mathrm{s}$ and $100 \mathrm{rpm}$ as same as the design condition of the previous section. The chord length of airfoil and radius of VAWT are also same with that of the design optimization problem, which results in the solidity of 0.21 in this VAWT rotor. The far-field boundary conditions are approximately same with that of the steady flow simulations. The unsteady flow simulations are performed for three rotations, and then the results of the last rotation are discussed in this section. The computational cost of this unsteady flow simulation is approximately 50 times more than that of the inexpensive steady flow approach explained in the previous section.

The flowfields around the optimal airfoil of D are shown in Fig.13. The distributions of the performance function 
$P$ of an airfoil blade in the unsteady simulations are compared in Fig.14 in the same manner as Fig.9. The x-axis is standardized by the rotational angle as Fig.9 by taking into account the airfoil position in the unsteady simulations. Qualitative agreement of the distributions can be observed between the steady inexpensive evaluations (Fig.9) and the unsteady evaluations (Fig.14). In detail, the peak performance in the range of $270 \leq \theta \leq 360$ degrees is well reproduced in the unsteady simulations, which typically corresponds to the airfoils of (ii)-c, (iii)-c, and (iv)-c in Fig.13. In this range of the rotational angle, assumptions of steady flow and of no flow interaction with the other two blades are satisfied reasonably well. Another peak performance in the range of $180 \leq \theta \leq 270$ degrees, which is observed in Fig.9, cannot be observed in the unsteady simulations although the performance change tendencies between the airfoil configurations are almost identical. This typically corresponds to the airfoil of (iv)-b, in which the uniform flow at this range is disturbed at its upstream by the precedent airfoil. The aerodynamic performance in this range is degenerated by this effect in the unsteady simulations. Large differences can be observed in the range of $15 \leq \theta \leq 90$ degrees where the instantaneous performance function values are negative in the all unsteady simulations. This is due to the effect of vortices that are generated in the downstream of the airfoil approximately at $\theta=0$ degree. The vortices are advected along with the blade airfoil, as observed around the airfoils of (i iii)-a, which makes some unsteady effects to the airfoil at the range of $15 \leq \theta \leq 90$ degrees. The averaged performance functions $\bar{P}$ obtained in these unsteady simulations are summarized in Fig.15 to compare with that obtained in the steady inexpensive evaluations. Although the performance function values degenerate in the unsteady simulations due to the effect of flow unsteadiness and interactions, it can be confirmed that relative performance changes are almost comparable between them. In addition, although different turbulence models were utilized in the steady / unsteady simulations, these results showed comparable performance, which indicates the robustness of the obtained optimal airfoil with respect to the change of the turbulence model. These results indicate the general validity of the obtained optimal configuration as well as the proposed design optimization approach via the steady inexpensive evaluations.

A general wind turbine performance function of power coefficient can be evaluated in the unsteady simulations by summing the torques acting on the three blade airfoils. The power coefficient is defined as follows:

$$
C_{P}=(T \omega) /\left(\frac{1}{2} \rho_{\infty} A U_{\infty}^{3}\right)
$$

where $T, \omega, \rho_{\infty}$ and $A$ are the time-averaged total torque of three blade airfoils, angular velocity, air density and swept area of VAWT, respectively. $A$ is set to $1.35 \mathrm{~m}$ in the present $2 \mathrm{D}$ simulations. The calculated power coefficient values are summarized in Fig.16, which again indicates the effectiveness of the present design optimization approach. The negative $C_{P}$ values in NACA and C airfoils imply the specified number of rotations is too large to achieve with the blade airfoils even at a free-rotating condition with no load.

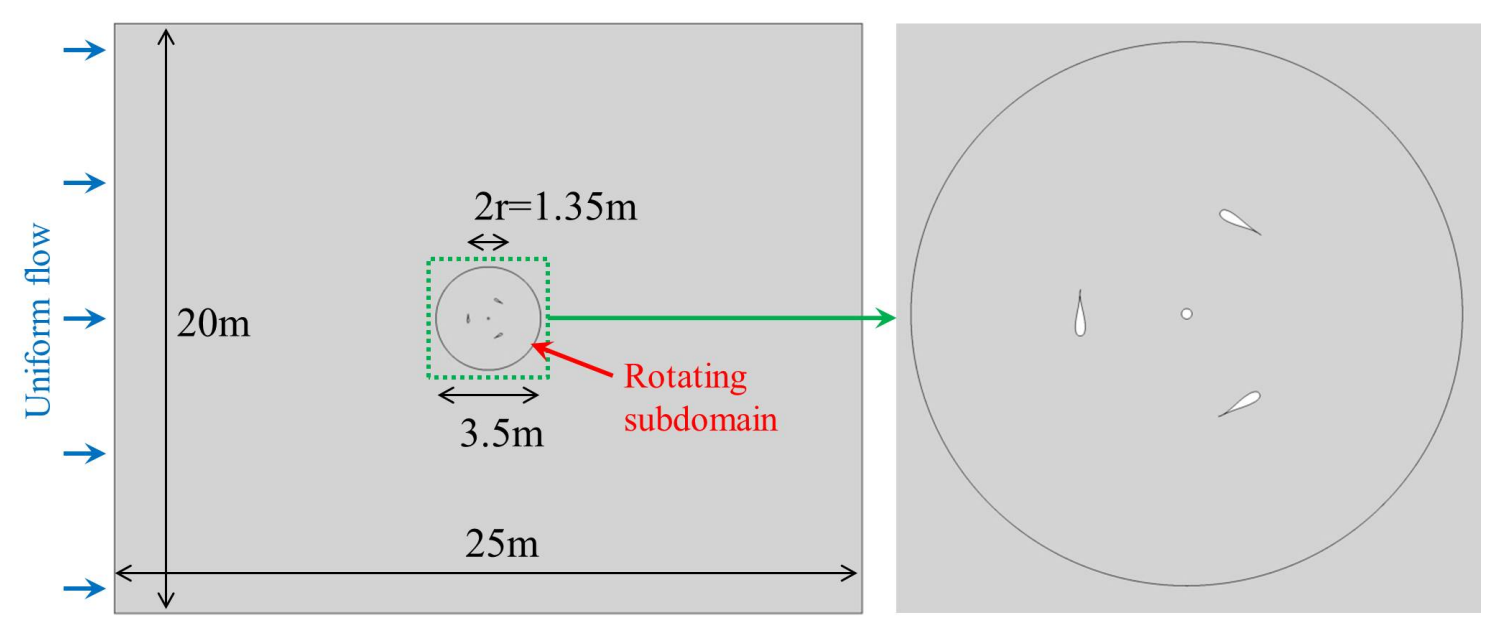

Fig.12 Definition of computational domain for 2D unsteady flow simulations. The central circle region is the rotating subdomain including VAWT. 


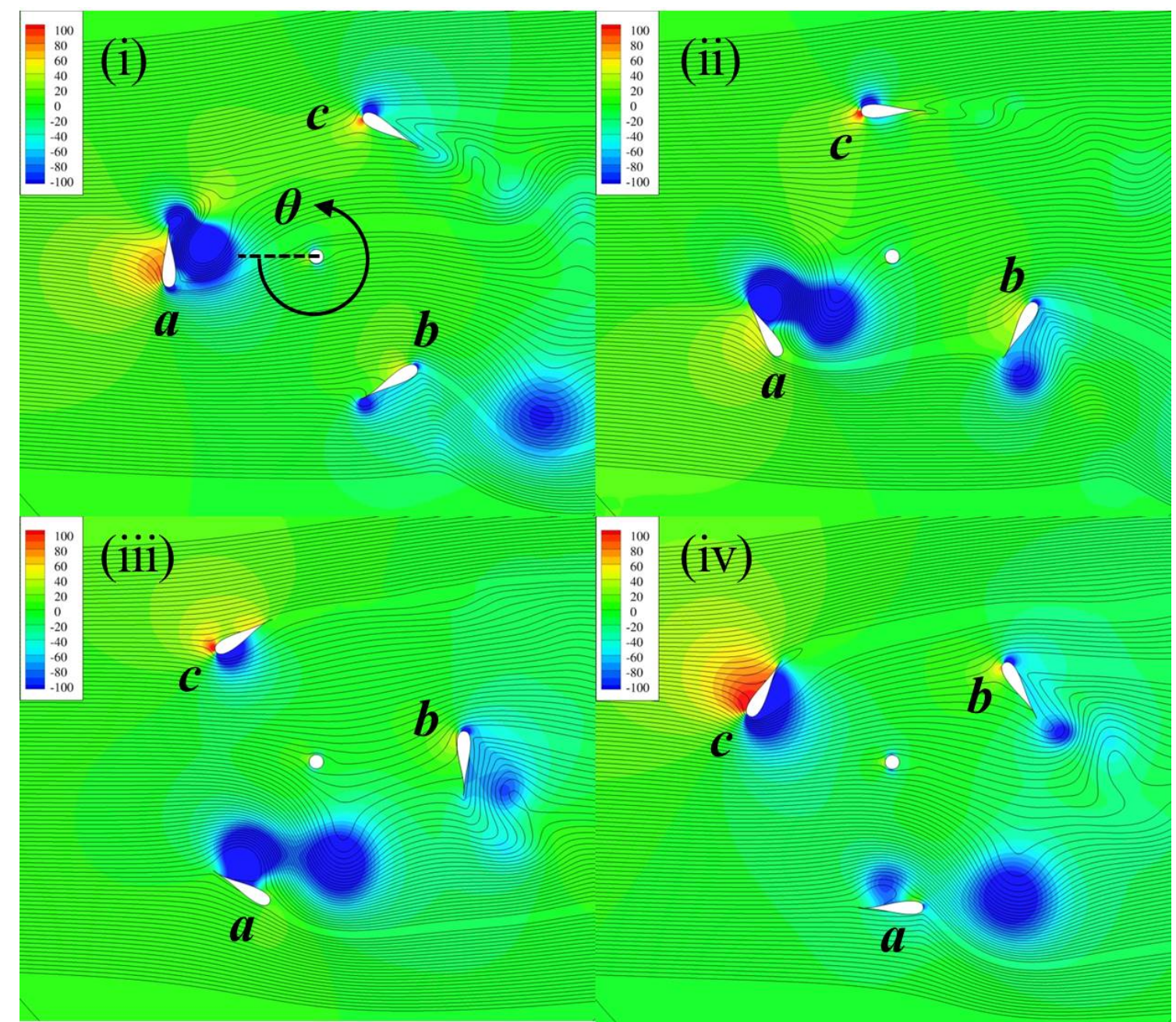

Fig.13 Two-dimensional unsteady flow evaluations of VAWT airfoil D. Instantaneous streamlines and pressure variation are visualized at several moments. The unit of pressure is the Pascal.

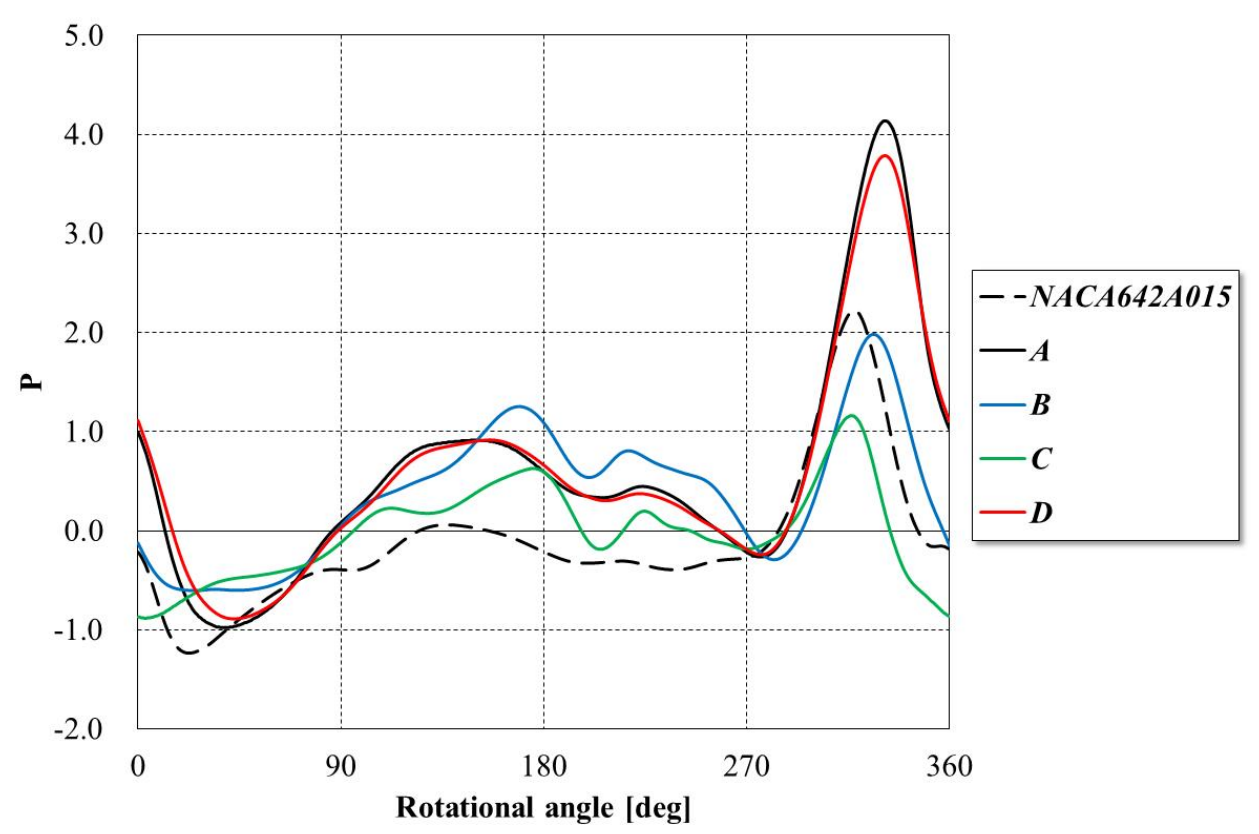

Fig.14 Distributions of performance function obtained in unsteady flow evaluations. This figure is arranged in the same manner as Fig.9. 


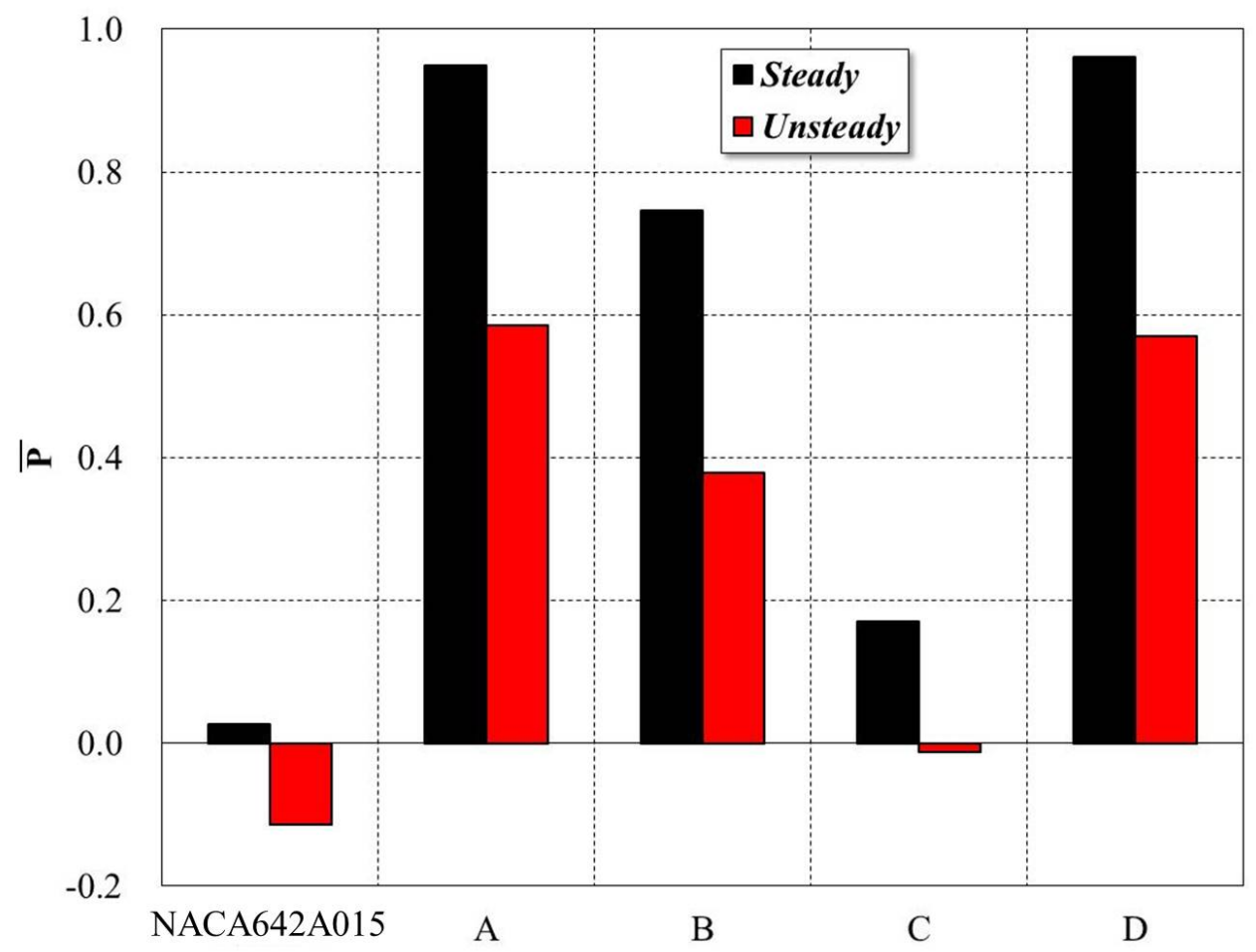

Fig.15 Comparison of averaged performance function between steady and unsteady simulations. Relative performance changes are comparable between steady and unsteady simulations.

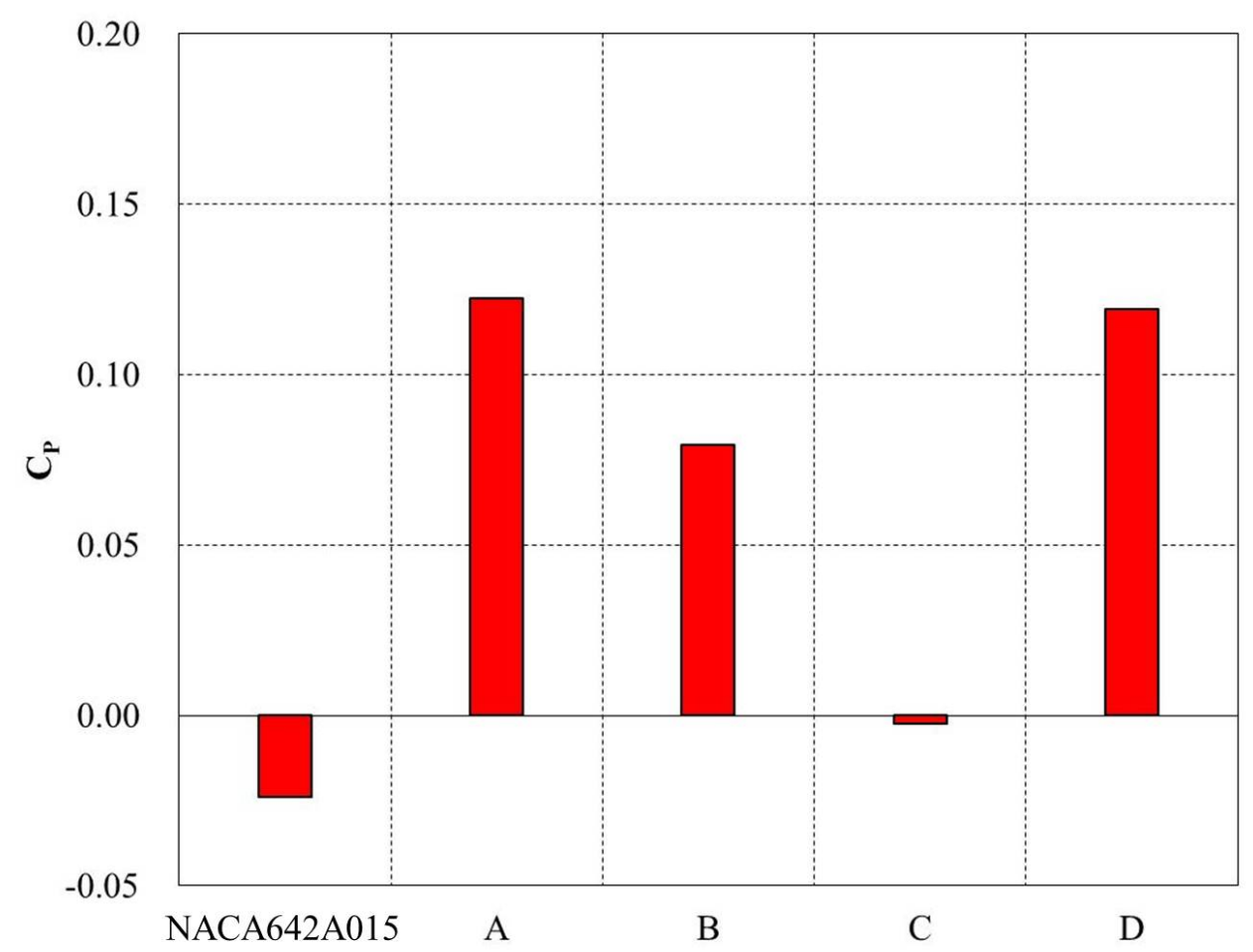

Fig.16 Comparison in power coefficient of representative optimal designs, that are evaluated from unsteady simulations.

\section{Conclusions}

In this paper, an efficient aerodynamic shape optimization of VAWT airfoil has been performed with a Kriging response surface model approach. Numerical evaluations of various airfoil shapes were treated inexpensively by considering the apparent flow velocity magnitudes and apparent angles of attack at multiple positions of VAWT airfoil. 
This numerical evaluation includes assumptions as two dimensional steady flow and no consideration about flow interactions between blade airfoils. The optimal VAWT airfoil has obtained by the maximization of a proposed objective function. The obtained optimal airfoil has achieved significant improvement in the aerodynamic performance compared with the NACA642A015 airfoil by suppressing flow separations at some rotational angles. The ANOVA analysis revealed the importance of the leading edge configuration in the present VAWT airfoil shape optimization. Finally, unsteady CFD simulations have been performed for the obtained optimal airfoil, which takes into account the flow interactions between three blade airfoils. The effectiveness of the proposed design optimization approach / obtained optimal airfoil configuration has been confirmed qualitatively by the investigations based on the unsteady simulations.

The one of major future research directions is additional validations of the optimal airfoil configuration by its experimental evaluations. We will also apply the developed optimization approach to different operational conditions of $\beta>1$. Moreover, the sophistication of the proposed design optimization problem is promising. Since the differences of the aerodynamic performance at some ranges of rotational angle have been confirmed between the steady/unsteady evaluations, some treatments for such range in the shape optimization problem will increase more the efficiency of the present shape optimization approach of VAWT airfoil.

\section{References}

Bah, E. A., Sankar, L., and Jagoda, J. Investigation on the use of multi-element airfoils for improving vertical axis wind turbine performance, AIAA Paper 2013-1109 (2013).

Carrigan, T. J., Dennis, B. H., Han, Z. X., and Wang, B. P., Aerodynamic shape optimization of a vertical-axis wind turbine using differential evolution, ISRN Renewable Energy Volume 2012, Article ID 528418 (2012).

Ferreira, C. S., Kuik, G. V., Bussel, G. V., and Scarano, F., Visualization by PIV of dynamic stall on a vertical axis wind turbine, Experiments in Fluids, Vol.46, Issue 1 (2009), pp.97-108.

Fonseca, C. M., and Fleming, P. J., Genetic algorithms for multiobjective optimization: formulation, discussion and generalization, Proceedings of the 5th International Conference on Genetic Algorithms, Morgan Kaufmann Publishers, Inc., San Mateo (1993), pp.416-423.

Forrester, A. I., Sobester, A., and Keane, A. J., Engineering design via surrogate modelling: a practical guide, John-Wiley and Sons (2008).

Greenblatt, D., Schulman, M., and Ben-Harav, A., Vertical axis wind turbine performance enhancement using plasma actuators, Renewable Energy, Vol.37, Issue 1 (2012), pp.345-354.

Han, Z. H., Zhang, K., Liu, J., and Song, W. P., Surrogate-based aerodynamic shape optimization with application to wind turbine airfoils, AIAA Paper 2013-1108 (2013).

Howell, R., Qin, N., Edwards, J., and Durrani, N., Wind tunnel and numerical study of a small vertical axis wind turbine, Renewable Energy, Vol.35, Issue 2 (2010), pp.412-422.

Jones, D. R., Schonlau, M., and Welch, W. J., Efficient global optimization of expensive black-box functions, Journal of Global Optimization, Vol.13, Issue 4 (1998), pp.455-492.

Kim, H. J., and Liou, M. S., New multi-objective genetic algorithms for diversity and convergence enhancement, AIAA Paper 2009-1168 (2009).

Seki, K., Research and development of high-performance airfoil sections for vertical axis wind turbine at low-Reynolds number, Transaction of JSME, Vol.57, No.536 (1991), pp.1297-1304 (in Japanese).

Wakashima, S., Miyashita, Y., Yamazaki, W., Yamada, N., Takahashi, T., and Otsuka, Y., On design and evaluation of performances of prototype Mg-alloy micro vertical axis wind turbine, Proceedings of 17th JSME Symposium on Power and Energy Systems (2012) (in Japanese).

Wakashima, S., Miyashita, Y., Yamada, N., Takahashi, T., Otsuka, Y., and Yamazaki, W., Numerical analysis of a rotating micro straight-wing VAWT model for engineering design educations, Transactions of the Japan Society of Mechanical Engineers Series C Vol.79, No.808 (2013), pp.2562-2566 (in Japanese).

Yamazaki, W., and Mavriplis, D. J., Derivative-enhanced variable fidelity surrogate modeling for aerodynamic functions, AIAA Journal, Vol.51, No.1 (2013), pp.126-137. 